\section{Tradução e validação para o Brasil da escala de imagem corporal para adolescentes - Offer Self-Image Questionnaire (OSIQ)}

\section{Translation and validation for Brazil of the body image scale for adolescents - Offer Self-Image Questionnaire (OSIQ)}

\section{Maria Aparecida Conti' \\ Norman Hearst" \\ Maria do Rosário Dias de Oliveira Latorre"'I}

'Programa de Transtorno Alimentar (AMBULIM) do Hospital das Clínicas da Faculdade de Medicina da Universidade de São Paulo (USP) - São Paulo (SP), Brasil

"Departamento de Epidemiologia e Bioestatística da Universidade da Califórnia em São Francisco - São Francisco (CA), EUA

"'Departamento de Epidemiologia da Faculdade de Saúde Pública da Universidade de São Paulo (USP) - São Paulo (SP), Brasil

Trabalho realizado na Faculdade de Saúde Pública da Universidade de São Paulo (USP) - São Paulo (SP), Brasil.

Fonte de financiamento: Conselho Nacional de Desenvolvimento Científico e Tecnológico (CNPq) - Processo no 140097/2005-8.

Correspondência: Maria Aparecida Conti - Instituto de Psiquiatria da USP - Programa de

Transtornos Alimentares (AMBULIM) - Av. Dr. Ovídio Pires de Campos, 785, 2ªndar -

CEP: 05403-010 - São Paulo (SP), Brasil - E-mail: maconti@usp.br

Conflito de interesse: nada a declarar.

\section{Resumo}

Objetivo: Apreciar a equivalência semântica e de mensuração da subescala de imagem corporal do Offer Self Image Questionnaire (OSIQ). Métodos: Participaram 386 adolescentes, na faixa etária de 10 a 18 anos, de ambos os sexos, de uma escola particular de ensino fundamental e médio. Realizou-se tradução, retrotradução, revisão técnica e avaliação do instrumento. Avaliou-se a consistência interna, a validade discriminante e concorrente e a confiabilidade. Resultados: $\mathrm{O}$ instrumento apresentou, para a consistência interna, valores que variaram de 0,43 a 0,51 e foi capaz de discriminar os grupos estudados - população total, meninos, meninas, e meninos em fase inicial de adolescência, segundo o estado nutricional $(\mathrm{p}<0,001 ; \mathrm{p}<0,009 ; \mathrm{p}=0,030 ; \mathrm{p}=0,043$, respectivamente). Na validade concorrente, houve correlação significativa entre algumas medidas antropométricas somente para o sexo feminino $(\mathrm{r}=-0,16$ e $\mathrm{p}=0,021$; $\mathrm{r}=-0,19$ e $\mathrm{p}=0,007)$; fase inicial $(\mathrm{r}=-0,23$ e $\mathrm{p}=0,008 ; \mathrm{r}=-0,26$ e $\mathrm{p}=0,003$ ) e intermediária $(\mathrm{r}=-0,29$ e $\mathrm{p}=0,010)$ e no reteste confirmou sua confiabilidade por meio da correlação intraclasse. Embora o instrumento tenha comprovado sua capacidade em discriminar os grupos estudados, de acordo com o estado nutricional, os demais resultados foram menos satisfatórios. Mais estudos são necessários para sua adaptação transcultural, com a aplicação de outras escalas comparativas. Conclusão: A subescala de imagem corporal do OSIQ encontra-se traduzida, no entanto, os resultados apresentados não são promissores, exigindo-se mais estudos.

Palavras-chave: estudos de validação; reprodutibilidade dos testes; tradução (processo); imagem corporal; adolescente; escalas. 


\section{Abstract}

Objective: To evaluate the semantic and measure equivalence of the body image sub-scale of the Offer Self Image Questionnaire (OSIQ). Methods: Participants were 386 teenagers, 10 to 18 years old, both sexes, enrolled in a private school (junior and high school age). Translation, back-translation, technique revision and evaluation were conducted. The Portuguese instrument was evaluated for internal consistency, discriminate and concurrent validity. Results: Internal consistency showed values from 0.43 to 0.54 and was able to discriminate all groups studied - the whole population, boys and girls, and boys in early adolescence, by nutritional status $(\mathrm{p}<0.001 ; \mathrm{p}<0.009 ; \mathrm{p}=0.030$; $\mathrm{p}=0.043$, respectively). Concurrent analyses showed significant correlation with anthropometric measures only for girls $(\mathrm{r}=-0.16$ and $\mathrm{p}=0.021 ; \mathrm{r}=-0.19$ and $\mathrm{p}=0.007)$, early adolescence $(\mathrm{r}=-0.23$ and $\mathrm{p}=0.008 ; \mathrm{r}=-0.26$ and $\mathrm{p}=0.003)$ and intermediate adolescence $(\mathrm{r}=-0.29$ and $\mathrm{p}=0.010)$ and the retest confirmed reliability by the coefficient of interclass correlation. Although the instrument has proven its ability to discriminate between the groups studied by nutritional state, other results were less satisfactory. More studies are necessary for full transcultural adaptation, including the application of other comparative scales. Conclusion: The body image sub-scale of the OSIQ was translated, but the results are not promising and require more studies.

Keywords: validation studies; reproducibility of results; translating; body image; adolescent; scales.

\section{Introdução}

A imagem corporal é a figura formada na mente do indivíduo acerca de seu tamanho, forma e estrutura corporais, envolvendo sentimentos relativos a essas características, bem como em relação às partes corporais constituintes ${ }^{1}$. Para Cash e Pruzinsky ${ }^{2}$, falar sobre imagem corporal é falar sobre "imagens corporais" devido às várias dimensões que a englobam. Consiste assim, no mínimo, em duas dimensões independentes: a perceptiva, relacionada ao julgamento do tamanho e forma corporais, e a atitudinal, relacionada ao afeto e à cognição ${ }^{2}$.

Por ser um construto multidimensional, várias técnicas de mensuração são passíveis de aplicação. De acordo com Thompson ${ }^{3}$, há, no mínimo, 50 medidas disponíveis para mensurar a imagem corporal, no entanto, um número reduzido de instrumentos recebeu a atenção devida com enfoque no processo de tradução, adaptação e validação para populações específicas.

Dos instrumentos disponíveis, os mais aplicados em estudos epidemiológicos são as escalas de silhueta e os questionários autoaplicáveis ${ }^{4}$. A escala de silhueta consiste em desenhos (silhuetas) de figuras humanas na qual o sujeito deve escolher duas imagens: uma que mais se aproxima de como se percebe e outra que se aproxime de como gostaria de ser. Essa escala avalia, exclusivamente, o grau de satisfação em relação à imagem corporal, sendo entendida como a diferença (valor) entre o corpo real - percebido e o ideal - desejado ${ }^{3}$. Já os questionários são compostos por questões de autopreenchimento na forma de escala likert de pontos, com variação para as respostas, normalmente de acordo com a frequência, concordância e/ou o grau de satisfação de um dado comportamento do indivíduo. São avaliados por meio de escores obtidos pelo cálculo da soma das respostas ${ }^{4}$.

Tanto as escalas de silhueta como os questionários autoaplicáveis são recursos bem vantajosos ${ }^{4}$, pois podem ser administrados com a mínima dificuldade. São práticos, visto que sua aplicação não exige 
especialistas treinados, a abordagem é coletiva, o que possibilita a participação de grandes amostras. Também há possibilidade de se inserir mais um instrumento no protocolo do estudo, sem comprometer o resultado final.

Optou-se, nesta pesquisa, pela escala de imagem corporal do Offer Self-Image Questionnaire $^{5}$ (OSIQ), que se trata de um teste de personalidade autodescritivo, estruturado a partir da teoria de personalidade psicodinâmica de desenvolvimento, como um método de avaliação abrangente acerca da consciência da autoimagem do adolescente $^{6}$. Foi desenvolvido nos Estados Unidos, entre os anos de 1961 e 1962, para avaliação de adolescentes sem distúrbios psíquicos.

Durante estes 40 anos de existência, o $\mathrm{OSIQ}^{5}$ foi aplicado em diferentes culturas, mostrando bons resultados psicométricos ${ }^{6-9}$.

No Brasil, ainda não existe a versão para o idioma português, bem como estudos de validade e reprodutibilidade. Devido à importância dessa escala em pesquisas relacionadas à imagem corporal, e por não existir sua versão para o idioma português, nem estudos que confirmem a sua acurácia, o presente trabalho objetivou apreciar a equivalência semântica e de mensuração da escala de imagem corporal para adolescentes do OSIQ.

\section{Métodos}

Este estudo baseou-se, parcialmente, nos procedimentos metodológicos de avaliação de questionários/instrumentos sugeridos por Herdman, Fox-Rushby e Badia ${ }^{10} \mathrm{e}$ aplicados por Hasselmann e Reichenheim ${ }^{11}$ e Grassi-Oliveira, Stein e Pezzi ${ }^{12}$. Duas etapas foram seguidas para análise dos diferentes tipos de equivalências: equivalência semântica (avaliação da transferência dos significados das palavras entre os diferentes idiomas) e equivalência de mensuração (avaliação das propriedades psicométricas dos instrumentos) ${ }^{11}$.

As avaliações da equivalência semântica ocorreram em 2006, de acordo com as seguintes fases: primeira, tradução do instrumento original em inglês para a língua portuguesa; segunda, retrotradução do instrumento em português para a língua inglesa; terceira, revisão técnica e avaliação da equivalência semântica.

A primeira fase envolveu a tradução do instrumento original do idioma inglês para o português, feita independentemente por duas pesquisadoras experientes e fluentes em inglês. Para a segunda etapa, as versões (traduções 1 e 2) foram novamente traduzidas para o inglês, por um professor nativo de língua inglesa. A etapa seguinte referiu-se à revisão técnica e à avaliação da equivalência semântica das versões e foi desenvolvida por duas profissionais (uma psicóloga e uma estatística), especialistas na área de adaptação de escalas. As discrepâncias foram ajustadas por meio da comparação das versões do instrumento - versão original (inglês); versão traduzida (português); versão retraduzida (inglês) e versão final (português). Desse modo, foi possível propor uma "versão-síntese" do instrumento que foi aplicada em um pré-teste, na escola selecionada, anterior à coleta de dados. Em seguida, avaliou-se a aceitação da versão do instrumento, chegando-se à versão final.

Para a equivalência de mensuração, é necessário avaliar as medidas de validade e reprodutibilidade, comparando-as com as encontradas no instrumento original ${ }^{11}$. Esta etapa do estudo iniciou-se com a coleta de dados em uma instituição da rede particular de Ensino Fundamental II e Médio, localizada no município de São Bernardo do Campo (SP), no ano de 2006.

Para o cálculo do tamanho da amostra, foram tomados por base os valores de $\alpha=5 \%$ e $\beta=10 \%$ para detectar um coeficiente de correlação de 0,40 a $0,60^{3,4}$, requerendo, assim, a participação mínima de 62 adolescentes de cada sexo.

Todos os adolescentes regularmente matriculados na instituição, cuja faixa etária era de 10 a 18 anos, foram convidados a participar do estudo. Dos adolescentes matriculados (466 alunos), 8 não quiseram 
participar, 5 não tiveram a autorização dos pais para participar e 65 não trouxeram o termo de consentimento livre e esclarecido. Vale lembrar que dois adolescentes foram excluídos, uma por motivo de gravidez e outro por estar fazendo uso de medicações psiquiátricas, o que poderia prejudicar a compreensão das questões. A amostra foi composta, ao final, por 386 adolescentes de ambos os sexos.

A análise das perdas (80 jovens) mostrou que não houve diferença em relação ao sexo ( $9 \%$ de perdas entre meninos e $8,2 \%$ entre meninas; $\mathrm{p}=0,331$ ) e à fase da adolescência $(4,3$ e $4,7 \%$ de perdas para os meninos em fase inicial e intermediária, respectivamente; $\mathrm{p}=0,899$; para as meninas, o valor correspondeu a 3,8 e $4,3 \%$ de perdas na fase inicial e intermediária, respectivamente; $\mathrm{p}=0,559$ ). Dessa forma, considerou-se que a amostra foi representativa da população estudada.

O questionário autoaplicável constava dos dados demográficos (idade, sexo, escolaridade) e da escala de autopreenchimento (teste). Foram coletadas medidas antropométricas (peso, estatura, circunferência do quadril, circunferência da cintura). A aplicação foi realizada em sala de aula, de forma coletiva, com orientações padronizadas. Após o preenchimento, o adolescente era encaminhado à sala de ginástica para a coleta das medidas antropométricas. Ao término dessa etapa, agendava-se o segundo encontro com todos os adolescentes participantes, o qual constou somente da reaplicação da escala de autopreenchimento (reteste), objetivando-se fazer as análises de reprodutibilidade do instrumento. Esse processo deu-se no intervalo de duas a três semanas ${ }^{4}$.

O questionário de imagem corporal é uma subescala do OSIQ ${ }^{5}$. Este é composto por 129 questões (12 escalas), sendo que 7 questões formam a escala de imagem corporal, na forma de autopreenchimento, em escala likert, com variação de 6 pontos: 1 (descreve-me muito bem); 2 (descreve-me bem); 3 (descreve-me mais ou menos); 4 (não me descreve); 5 (não me descreve de verdade); 6 (não me descreve em nada). Lindfors et al. ${ }^{6}$ utilizaram um escore por meio do somatório das questões, revertendo os valores das pontuações das questões com direção negativa, a saber: 1, 2, 4 e 5 . Os novos valores passaram a ser ( $1=6),(2=5),(3=4),(4=3),(5=2)$, $(6=1)$. Esse escore varia de 7 a 42 pontos, sendo que alto escore é representativo de autoimagem positiva.

No que se refere aos fatores estruturantes do OSIQ ${ }^{5}$, não há um consenso, sendo este tema ainda pesquisado ${ }^{6}$. Em estudo prévio, Offer et al. ${ }^{5}$ agruparam as escalas em cinco dimensões, a saber: psicológica, social, sexual, familiar e enfrentamento. Já Lindfors et al. ${ }^{6}$ registraram outros domínios: ansiedade pessoal, relacionamento social, atitudes sexuais, relacionamento familiar e consciência social, sendo que a escala de imagem corporal integra o primeiro domínio.

Cabe esclarecer que, para Lindfors et al. ${ }^{6}$, a aplicação de uma escala separadamente das demais escalas que compõem o instrumento geral, não é uma prática indicada, pois o OSIQ ${ }^{5}$ é um instrumento multidimensional, enfraquecendo seu poder de análise e diagnóstico. No entanto, não há um consenso na área, havendo pesquisadores que optam, de acordo com seu objetivo, pelo uso de escalas isoladamente. Esta escolha pode ser observada no estudo de PolceLynch et al. ${ }^{13}$, que aplicaram três das escalas do OSIQ (imagem corporal, relacionamento entre amigos e relacionamento familiar), analisando-as separadamente. Thompson ${ }^{3}$ reforça a necessidade da adequação dos instrumentos por parte dos pesquisadores, em relação a seus objetivos. Devido a esses fatores, optou-se pela escolha da escala de imagem corporal do OSIQ, pois esta é específica para avaliar o aspecto perceptivo da imagem corporal em adolescentes.

Para avaliar a clareza e o grau de compreensão da escala na íntegra, foi solicitado aos adolescentes que respondessem a uma escala verbal-numérica adaptada ${ }^{12}$ ao término da aplicação do instrumento, por meio da seguinte pergunta: "Você 
entendeu o que foi perguntado nesta escala?". As respostas eram do tipo escala likert: 0 (não entendi nada); 1 (entendi um pouco); 2 (entendi mais ou menos); 3 (entendi quase tudo, mas tive algumas dúvidas); 4 (entendi perfeitamente e não tenho dúvidas). Conforme GrassiOliveira Stein e Pezzi ${ }^{12}$, foi estabelecido que as respostas 0 , 1 e 2 seriam consideradas como indicadores de uma compreensão insuficiente.

A avaliação antropométrica foi realizada pela primeira autora da presente pesquisa com aferição do peso, estatura corporal, circunferência do quadril e cintura dos alunos. Para a medição de peso corporal, foi utilizada uma balança eletrônica do tipo plataforma, com capacidade para 150 $\mathrm{kg}$ e graduação em $100 \mathrm{~g}$, com os adolescentes trajando roupas leves e descalços ${ }^{14}$. Para a estatura, utilizou-se o estadiômetro portátil (SECA) fixado à parede com escala em milímetros ( $\mathrm{mm}$ ). Solicitou-se ao adolescente que encostasse à parede os calcanhares, a panturrilha, os glúteos e os ombros e que a cabeça ficasse posicionada no plano de Frankfurt ${ }^{14}$. Para medição das circunferências da cintura, foi utilizada uma fita métrica que foi passada, firmemente, ao redor da cintura, no ponto médio entre a última costela e a crista ilíaca, e para o quadril, na extensão posterior máxima, ao redor dos glúteos ${ }^{15}$. Para todas as medidas, foram realizadas duas mensurações e foi considerada a média dos valores obtidos.

A classificação do estado nutricional dos adolescentes foi feita segundo recomendação da Organização Mundial da Saúde $(\mathrm{OMS})^{16}$ para essa faixa etária: magreza $<$ percentil 5; peso normal entre o percentil $5 \mathrm{e}$ $<$ percentil 85 ; risco para sobrepeso $\geq$ percentil 85 e $<$ percentil 90; obeso $\geq$ percentil 90.

Foi realizada análise estatística descritiva e calculados a média, os desvios padrão, os valores mínimos e máximos para as variáveis antropométricas, demográficas e idade. As propriedades psicométricas foram verificadas pela consistência interna, apreciação da validade discriminante e concorrente, confiabilidade, tempo de conclusão e compreensão verbal da escala. As análises foram realizadas para a população total, meninos e meninas, e fase da adolescência: Inicial ( 10 a 14 anos) e Intermediária $(15 \text { a } 18 \text { anos })^{16}$, respectivamente.

Avaliou-se a consistência interna da escala por meio do cálculo do coeficiente $\alpha$ de Cronbach. Na apreciação da validade discriminante, foram comparados quatro grupos: magro, peso normal, sobrepeso e obeso $^{16}$, por meio da análise de variância de Kruskal Wallis. Espera-se que os adolescentes com obesidade expressem maior insatisfação quando comparados aos demais. Para apreciação da validade concorrente, inicialmente, foram testadas as distribuições das variáveis, e, por não atender a condição de normalidade, calculou-se o coeficiente de correlação de Spearman entre o escore da escala e o índice de massa corporal (IMC), a circunferência da cintura (CC) e a razão cintura/quadril (RCQ), respectivamente. Espera-se o seguinte resultado: quanto maior o IMC, a CC ou a RCQ, maior será o grau de insatisfação.

A confiabilidade foi avaliada pelo gráfico de Bland-Altman ${ }^{17}$ e pela comparação das médias dos escores da escala entre a primeira aplicação (teste), e a segunda aplicação (reteste), utilizando o teste de Wilcoxon, o coeficiente de correlação intraclasse $\left(\mathrm{r}_{\text {intra- }}\right.$ classe). Para análise do tempo de conclusão da escala, registrou-se o tempo médio utilizado pelo adolescente para o preenchimento da mesma. Vale lembrar que a compreensão verbal foi analisada pelos valores médios.

Os adolescentes foram convidados a participar de forma voluntária e foi solicitado que entregassem aos pais o termo de consentimento livre e esclarecido e o trouxessem devidamente assinado. Somente nessas condições, iniciou-se a coleta de dados.

O presente estudo está de acordo com as normas no 196 , de 10/10/1996, do Conselho Nacional de Saúde, e foi aprovado pelo Comitê de Ética e Pesquisa da Faculdade de Saúde Pública da Universidade de São Paulo (COEP 233/05). 


\section{Resultados}

\section{Caracterização da amostra e descrição da escala de Imagem Corporal do OSIQ}

Participaram da pesquisa 386 adolescentes, sendo que $46,1 \%$ (178) e 53,9\% (208) são do sexo masculino e feminino, respectivamente. Desses, 261 (67,6\%) estavam no Ensino Fundamental (5a a $8^{\mathrm{a}}$ série) e 125 (32,4\%) no Ensino Médio; 175 (45,3\%) e 211 $(54,7 \%)$, na faixa etária de 10 a 14 anos e 15 a 18 anos, respectivamente.

Foram encontrados para peso, estatura e IMC valores médios (desvios padrão) de 59,3 $\mathrm{kg}(16,4 \mathrm{~kg}), 163,8 \mathrm{~cm}(11,7 \mathrm{~cm})$, $21,9 \mathrm{~kg} / \mathrm{cm}^{2}\left(4,6 \mathrm{~kg} / \mathrm{cm}^{2}\right)$ e $55,8 \mathrm{~kg}(11,5 \mathrm{~kg})$, $158,7 \mathrm{~cm}(7,5 \mathrm{~cm}), 22,0 \mathrm{~kg} / \mathrm{cm}^{2}\left(3,7 \mathrm{~kg} / \mathrm{cm}^{2}\right)$, para meninos e meninas, respectivamente, de acordo com a Tabela 1 .

As questões que descrevem uma imagem corporal negativa por meio da resposta "descreve-me muito bem" obtiveram maior porcentagem para a questão número 3 (No ano passado, fiquei muito preocupado(a) a respeito de minha saúde) com 12,4\% e a número 7 (Quando os outros me observam, devem achar que sou pouco desenvolvido) com 10,1\%. Já a questão número 4 (Tenho orgulho do meu corpo), com $7 \%$ dos adolescentes afirmando que "não me descreve em nada", implicou, assim, uma relação mais negativa com a imagem corporal, de acordo com a Tabela 2.

\section{Equivalência semântica}

Na tradução do instrumento, não houve necessidade de adaptação de palavras e/ou expressões por se tratar de sinônimos, fato que facilitou o processo de adaptação transcultural. Isto pode ser observado na questão 3, com a expressão In the past year, retraduzida como Overing the past year. O mesmo para a questão 6, com a palavra frequently, que foi retraduzida como often. Já na questão 7, na retradução, foi inserido o pronome that, não presente na versão original. E nas opções de reposta, no item 3, a palavra fairly well foi retraduzida por little. Essas nuanças podem ser observadas na Tabela 3.

O pré-teste foi realizado com 4 jovens, de ambos os sexos, sendo 2 do Ensino Fundamental (12 anos) e 2 do Ensino Médio (16 anos). A aplicação transcorreu sem nenhum questionamento, o que demonstrou que o instrumento seria de fácil compreensão.

\section{Equivalência de mensuração - caracterís- ticas psicométricas da escala}

$\mathrm{Na}$ análise da consistência interna, foram registrados valores do coeficiente $\alpha$ de Cronbach de 0,51 para população total e 0,53 e 0,45 para meninos e meninas, respectivamente (Tabela 4). Já em relação à fase da adolescência, os valores variaram

Tabela 1. Estatística descritiva dos adolescentes. São Bernardo do Campo (SP), 2006

Table 1. Descriptive statistics of adolescents. São Bernardo do Campo (SP), 2006

\begin{tabular}{|c|c|c|c|c|c|c|c|c|c|}
\hline \multirow[b]{2}{*}{ Variável } & \multicolumn{3}{|c|}{ Total } & \multicolumn{3}{|c|}{ Meninas } & \multicolumn{3}{|c|}{ Meninos } \\
\hline & Média & $\begin{array}{l}\text { Desvio } \\
\text { padrão }\end{array}$ & $\begin{array}{l}\text { Mínimo- } \\
\text { máximo }\end{array}$ & Média & $\begin{array}{l}\text { Desvio } \\
\text { padrão }\end{array}$ & $\begin{array}{l}\text { Mínimo- } \\
\text { máximo }\end{array}$ & Média & $\begin{array}{l}\text { Desvio } \\
\text { padrão }\end{array}$ & $\begin{array}{l}\text { Mínimo- } \\
\text { máximo }\end{array}$ \\
\hline Idade (anos) & 13,8 & 2,1 & $11-18$ & 13,9 & 2,2 & $11-18$ & 13,8 & 2,0 & $11-18$ \\
\hline Peso (kg) & 57,5 & 14,0 & $26-116$ & 72,9 & 8,8 & $58,6-103,6$ & 59,3 & 3,4 & $30,5-115,3$ \\
\hline Estatura (cm) & 161,1 & 10,0 & $128-194$ & 158,7 & 7,5 & $127,7-176,8$ & 163,8 & 8,8 & $113,4-194,2$ \\
\hline $\begin{array}{l}\text { Circunferência da } \\
\text { cintura }(\mathrm{cm})\end{array}$ & 74,2 & 10,2 & $57,3-120,2$ & 72,9 & 8,8 & $58,6-103,6$ & 75,7 & 11,5 & $57,3-120,2$ \\
\hline $\begin{array}{l}\text { Circunferência do } \\
\text { quadril }(\mathrm{cm})\end{array}$ & 90,5 & 9,6 & $65,3-127,6$ & 91,9 & 8,8 & $65,3-118,3$ & 89,0 & 10,2 & $68,6-127,6$ \\
\hline $\begin{array}{l}\text { Razão cintura/ } \\
\text { quadril }\end{array}$ & 0,8 & 0,1 & $07-1,2$ & 0,8 & 0,1 & $0,7-1,2$ & 0,8 & 0,1 & $0,7-1,1$ \\
\hline $\begin{array}{l}\text { Índice de massa } \\
\text { corporal }\left(\mathrm{kg} / \mathrm{cm}^{2}\right)\end{array}$ & 22,0 & 4,1 & $15-45$ & 22,0 & 3,7 & $15,7-38,7$ & 21,9 & 4,6 & $14,7-44,7$ \\
\hline
\end{tabular}


Tabela 2. Número e porcentagem de adolescentes, segundo as questões que compõem a subescala de imagem corporal do OSIQ. Momento 1. São Bernardo do Campo (SP), 2006

Table 2. Numbers and percentages of adolescents' responses to questions in body image subscale of OSIQ. Time 1. São Bernardo do Campo (SP), 2006

\begin{tabular}{|c|c|c|c|c|c|c|c|}
\hline \multirow{3}{*}{ Questões } & \multicolumn{6}{|c|}{ Respostas } & \multirow[b]{2}{*}{ Total } \\
\hline & $\begin{array}{l}\text { Descreve-me } \\
\text { muito bem }\end{array}$ & $\begin{array}{l}\text { Descreve-me } \\
\text { bem }\end{array}$ & $\begin{array}{l}\text { Descreve-me } \\
\text { mais ou menos }\end{array}$ & $\begin{array}{l}\text { Não me } \\
\text { descreve }\end{array}$ & $\begin{array}{l}\text { Não me } \\
\text { descreve de } \\
\text { verdade }\end{array}$ & $\begin{array}{l}\text { Não me } \\
\text { descreve } \\
\text { em nada }\end{array}$ & \\
\hline & n (\%) & $\mathrm{n}(\%)$ & $\mathrm{n}(\%)$ & $\mathrm{n}(\%)$ & $\mathrm{n}(\%)$ & $\mathrm{n}(\%)$ & $\mathrm{n}(\%)$ \\
\hline $\begin{array}{l}1 \text { - As mudanças recentes } \\
\text { em meu corpo me dão } \\
\text { alguma satisfação. }\end{array}$ & $99(25,6)$ & $114(29,5)$ & $129(33,4)$ & $25(6,5)$ & $4(1,0)$ & $15(3,9)$ & $386(100)$ \\
\hline $\begin{array}{l}2 \text { - A imagem que faço de } \\
\text { mim no futuro me satisfaz. }\end{array}$ & $179(46,4)$ & $119(30,8)$ & $59(15,3)$ & $16(4,1)$ & $5(1,3)$ & $8(2,1)$ & $386(100)$ \\
\hline $\begin{array}{l}3 \text { - No ano passado, fiquei } \\
\text { muito preocupado (a) a } \\
\text { respeito de minha saúde. }\end{array}$ & $48(12,4)$ & $51(13,2)$ & $45(11,7)$ & $68(17,6)$ & $25(6,5)$ & $149(38,6)$ & $386(100)$ \\
\hline $\begin{array}{l}4 \text { - Tenho orgulho do meu } \\
\text { corpo. }\end{array}$ & $104(26,9)$ & $76(19,7)$ & $105(27,2)$ & $57(14,8)$ & $17(4,4)$ & $27(7,0)$ & $386(100)$ \\
\hline $\begin{array}{l}5 \text { - Eu me sinto forte e } \\
\text { saudável. }\end{array}$ & $150(38,9)$ & $112(29,0)$ & $79(20,5)$ & $29(7,5)$ & $10(2,6)$ & $6(1,6)$ & $386(100)$ \\
\hline $\begin{array}{l}6 \text { - Eu, frequentemente, } \\
\text { sinto-me feio (a) e sem } \\
\text { atrativos }\end{array}$ & $33(8,5)$ & $40(10,4)$ & $86(22,3)$ & $49(12,7)$ & $45(11,7)$ & $133(34,5)$ & $386(100)$ \\
\hline $\begin{array}{l}7 \text { - Quando os outros me } \\
\text { observam, devem pensar } \\
\text { que sou pouco desen- } \\
\text { volvido (a). }\end{array}$ & $39(10,1)$ & $50(13,0)$ & $59(15,3)$ & $56(14,5)$ & $42(10,9)$ & $140(36,3)$ & $386(100)$ \\
\hline
\end{tabular}

Fonte: Offer Self-Image Questionnaire (OSIQ).

Source:Offer Self-Image Questionnaire (OSIQ).

entre 0,43 (fase inicial do sexo feminino) a 0,54 (fase final do sexo masculino), conforme Tabela 5. Na validade discriminante, houve diferença estatisticamente significativa nas médias dos escores entre os quatro subgrupos estudados para a população total $(\mathrm{p}<0,001)$, meninos $(\mathrm{p}<0,009) \mathrm{e}$ meninas $(p<0,030)$, e fase da adolescência para sexo masculino $(\mathrm{p}=0,043)$. Na análise da validade concorrente, registrou-se para a população total $(\mathrm{r}=-0,15 \mathrm{e} \mathrm{p}<0,003$; $\mathrm{r}=-0,01$ e $\mathrm{p}=0,781 ; \mathrm{r}=-0,11$ e $\mathrm{p}<0,033)$, meninos $(r=-0,08$ e $\mathrm{p}=0,313 ; \mathrm{r}=-0,17$ e $\mathrm{p}=0,020 ; \mathrm{r}=-0,08$ e $\mathrm{p}=0,243)$ e meninas $(\mathrm{r}=-0,16$ e $\mathrm{p}=0,021 ; \mathrm{r}=-0,15$ e $\mathrm{p}=0,031$; $\mathrm{r}=-0,19$ e $\mathrm{p}=0,007$ ), correlações estatisticamente significativas entre o IMC, a RCQ e a CC, respectivamente. Observa-se que, na correlação entre a RCQ para a população total, e IMC e CC para os meninos, não foram obtidos resultados estatisticamente significativos (Tabela 4). Já ao se observarem as fases da adolescência, somente para as meninas em fase inicial $(\mathrm{r}=-0,23 \mathrm{e}$ $\mathrm{p}=0,008 ; \mathrm{r}=-0,26$ e $\mathrm{p}=0,003)$ e final $(\mathrm{r}=-0,20$ $\mathrm{e} p=0,010)$, foram registradas correlações estatisticamente significativas entre IMC, CC, RCQ, respectivamente (Tabela 5).

Na confiabilidade, para a população total: 31,2 (5,2); 31,8 (5,9) p=0,012; e meninos: 32,6 $(5,2) ; 33,3(5,7) \mathrm{p}=0,034$; houve diferença estatisticamente significativa nas médias (desvios padrão) dos escores, quando foram considerados os momentos $1 \mathrm{e} 2$, respectivamente. O mesmo não ocorreu com as meninas: $30,1(5,0) ; 30,4(5,8) ; \mathrm{p}=0,153$. Já quando foram consideradas as fases da adolescência, para as meninas, na fase inicial: $33,2(4,6)$; $33,9(5,1) \mathrm{p}=0,198$; e para os meninos, na fase final: 30,1 (5,1); 30,1 (6,1) p=0,829; não houve diferença estatisticamente significativa nas médias dos escores (Tabela 5). 
Tabela 3. Avaliação da equivalência semântica do OSIQ: subescala original (inglês), versão traduzida (português), versão retraduzida (inglês) e versão final (português). São Bernardo do Campo (SP), 2006

Table 3. Evaluation of semantic equivalence of OSIQ: original subscale (English), translated version (Portuguese), back-translated version (English), and final version (English). São Bernardo do Campo (SP), 2006

\begin{tabular}{|c|c|c|c|}
\hline Documento original & Versão traduzida & Versão retraduzida & Versão final \\
\hline $\begin{array}{l}\text { Offer Self-Image Questionnaire } \\
\text { (OSIQ) }\end{array}$ & $\begin{array}{l}\text { Questionário de } \\
\text { autoimagem }\end{array}$ & Self-Image Questionnaire & $\begin{array}{l}\text { Questionário de Imagem } \\
\text { Corporal }\end{array}$ \\
\hline $\begin{array}{l}\text { 1. The recent changes in my } \\
\text { body have given me some } \\
\text { satisfaction. }\end{array}$ & $\begin{array}{l}\text { As mudanças recentes } \\
\text { em meu corpo me dão } \\
\text { alguma satisfação. }\end{array}$ & $\begin{array}{l}\text { Recent changes in my body } \\
\text { give me some satisfaction. }\end{array}$ & $\begin{array}{l}\text { As mudanças recentes em } \\
\text { meu corpo me dão alguma } \\
\text { satisfação. }\end{array}$ \\
\hline $\begin{array}{l}\text { 2. The picture I have of myself } \\
\text { in the future satisfies me. }\end{array}$ & $\begin{array}{l}\text { A imagem que faço } \\
\text { de mim no futuro me } \\
\text { satisfaz. }\end{array}$ & $\begin{array}{l}\text { The image I have of myself } \\
\text { in the future satisfies me. }\end{array}$ & $\begin{array}{l}\text { A imagem que faço de mim no } \\
\text { futuro me satisfaz. }\end{array}$ \\
\hline $\begin{array}{l}\text { 3. In the past year I have } \\
\text { been very worried about my } \\
\text { health. }\end{array}$ & $\begin{array}{l}\text { No ano passado, fiquei } \\
\text { muito(a) preocupado(a) a } \\
\text { respeito de minha saúde. }\end{array}$ & $\begin{array}{l}\text { Overing the past year, I } \\
\text { was very worried about my } \\
\text { health. }\end{array}$ & $\begin{array}{l}\text { No ano passado, fiquei } \\
\text { muito(a) preocupado(a) a } \\
\text { respeito de minha saúde. }\end{array}$ \\
\hline 4. I am proud of my body. & $\begin{array}{l}\text { Tenho orgulho do meu } \\
\text { corpo. }\end{array}$ & I am proud of my body & Tenho orgulho do meu corpo. \\
\hline 5. I feel strong and healthy. & $\begin{array}{l}\text { Eu me sinto forte e } \\
\text { saudável. }\end{array}$ & I feel stro & Eu me sinto forte e saudável. \\
\hline $\begin{array}{l}\text { 6. I frequently feel ugly and } \\
\text { unattractive. }\end{array}$ & $\begin{array}{l}\text { Eu, frequentemente, } \\
\text { sinto-me feio(a) e sem } \\
\text { atrativos. }\end{array}$ & $\begin{array}{l}\text { I often feel ugly and } \\
\text { unattractive }\end{array}$ & $\begin{array}{l}\text { Eu frequentemente sinto-me } \\
\text { feio(a) e sem atrativos. }\end{array}$ \\
\hline $\begin{array}{l}\text { 7. When others look at me } \\
\text { they must think I am poorly } \\
\text { developed. }\end{array}$ & $\begin{array}{l}\text { Quando os outros } \\
\text { me observam, devem } \\
\text { pensar que sou pouco } \\
\text { desenvolvido. }\end{array}$ & $\begin{array}{l}\text { When others look at me, } \\
\text { they must think that I am } \\
\text { poorly developed. }\end{array}$ & $\begin{array}{l}\text { Quando os outros me } \\
\text { observam, devem pensar que } \\
\text { sou pouco desenvolvido. }\end{array}$ \\
\hline 1. describes me very well & 1. descreve-me muito bem & 1. describes me very well & 1. descreve-me muito bem \\
\hline 2. describe me well & 2. descreve-me bem & 2. describe me well & 2. descreve-me bem \\
\hline 3. describe me fairly well & 3. descreve-me mais ou & 3. describe me a little & 3. descreve-me mais ou menos \\
\hline $\begin{array}{l}\text { 4. does not describe me } \\
\text { 5. does not really describe me }\end{array}$ & $\begin{array}{l}\text { menos } \\
\text { 4. não me descreve }\end{array}$ & $\begin{array}{l}\text { 4. does not describe me } \\
\text { 5. does not fully describe me }\end{array}$ & $\begin{array}{l}\text { 4. não me descreve } \\
\text { 5. não me descreve de verdade }\end{array}$ \\
\hline 6. does not describe me at all & $\begin{array}{l}\text { 5. não me descreve de } \\
\text { verdade } \\
\text { 6. não me descreve em } \\
\text { nada }\end{array}$ & 6. does not describe me at all & 6. não me descreve em nada \\
\hline
\end{tabular}

Fonte: Offer Self-Image Questionnaire (OSIQ).

Source:Offer Self-Image Questionnaire (OSIQ).

Os coeficientes de correlação intraclasse entre os escores dos momentos 1 e 2 foram significativos para a população total $\left(\mathrm{r}_{\text {icc }}=0,63 \mathrm{p}<0,001\right)$, meninos $\left(\mathrm{r}_{\text {icc }}=0,59\right.$ $\mathrm{p}<0,001)$ e meninas $\left(\mathrm{r}_{\text {icc }}=0,63 \mathrm{p}<0,001\right)$ (Tabela 4). O mesmo pode ser observado em relação às fases inicial $\left(\mathrm{r}_{\mathrm{icc}}=0,65 \mathrm{p}<0,001\right.$; $\mathrm{r}_{\text {icc }}=0,72 ; \mathrm{p}<0,001$ e intermediária $\left(\mathrm{r}_{\mathrm{icc}}=0,45\right.$ $\left.\mathrm{p}<0,001 ; \mathrm{r}_{\text {icc }}=0,46 \mathrm{p}<0,001\right)$ da adolescência, para o sexo masculino e feminino, respectivamente, segundo dados da Tabela 5 .
Na Figura 1, observa-se que a subescala de imagem corporal do OSIQ apresentou boa distribuição aleatória ao redor do zero, com poucos pontos fora do limite para os grupos estudados (fase inicial e intermediária da adolescência para os sexos masculino e feminino).

O tempo médio de conclusão da escala foi inferior a 2 minutos e a compreensão verbal correspondeu a valores médios de 3,3 minutos (máximo 4) (Tabelas 4 e 5). 
Tabela 4. Resumo das evidências das análises de validade e confiabilidade da subescala de imagem corporal do OSIQ para a população total, meninos e meninas. São Bernardo do Campo (SP), 2006

Table 4. Summary of findings of validity and reliability of body image subscale of OSIQ for entire study population, male and female. São Bernardo do Campo (SP), 2006

\begin{tabular}{|c|c|c|c|c|c|c|c|}
\hline Análises & Parâmetro & $\begin{array}{c}\text { População } \\
\text { total }\end{array}$ & $\mathrm{p}$ & Masculino & $\mathrm{p}$ & Feminino & $\mathrm{p}$ \\
\hline Validade discriminante & média (dp) & & & & & & \\
\hline magreza & & $33,1(6,7)$ & $<0,001$ & $34,7(5,8)$ & $<0,009$ & $31,7(7,5)$ & 0,030 \\
\hline peso normal & & $31,6(5,0)$ & & $33,1(4,9)$ & & $30,4(4,7)$ & \\
\hline sobrepeso & & $28,8(5,4)$ & & $29,8(5,9)$ & & $28,0(5,1)$ & \\
\hline obeso & & $28,0(5,4)$ & & $29,2(5,5)$ & & $26,6(5,2)$ & \\
\hline Validade concorrente & $r(p)$ & & & & & & \\
\hline IMC & & $-0,15$ & 0,003 & $-0,08$ & 0,313 & $-0,16$ & 0,021 \\
\hline $\mathrm{RCQ}$ & & $-0,01$ & 0,781 & $-0,17$ & 0,020 & $-0,15$ & 0,031 \\
\hline $\mathrm{CC}$ & & $-0,11$ & 0,033 & $-0,08$ & 0,243 & $-0,19$ & 0,007 \\
\hline Consistência interna & $\alpha$ Cronbach & 0,51 & & 0,53 & & 0,45 & \\
\hline \multirow[t]{3}{*}{ Confiabilidade } & média (dp) & $31,22(5,25)^{\mathrm{M} 1}$ & 0,012 & $32,6(5,2)^{\mathrm{M} 1}$ & 0,034 & $30,1(5,0)^{\mathrm{M} 1}$ & 0,153 \\
\hline & & $31,77(5,91)^{\mathrm{M} 2}$ & & $33,3(5,7)^{\mathrm{M} 2}$ & & $30,4(5,8)^{\mathrm{M} 2}$ & \\
\hline & $r_{j s c}(p)$ & 0,63 & $<0,001$ & 0,59 & $<0,001$ & 0,63 & $<0,001$ \\
\hline Tempo (segundos) & média (dp) & $90(50)$ & & $90(60)$ & & $90(50)$ & \\
\hline Compreensão verbal & média (dp) & $3,3(1,0)$ & & $3,2(1,0)$ & & $3,3(0,9)$ & \\
\hline
\end{tabular}

IMC: índice de massa corporal; RCQ: razão circunferência cintura/quadril; CC: circunferência da cintura, (dp): desvio padrão; $r$ (p): correlação; $r_{\text {icc }}$ (p): correlação

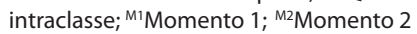

IMC: body mass index; RCQ: waist/hip circumference ratio; CC: waist circumference; (dp) standard deviation: $r$ ( $p)$ : correlation; $r_{\text {icc }}(p)$ : interclass correlation coeff-

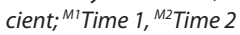

Tabela 5. Resumo das evidências das análises de validade e confiabilidade da subescala de imagem corporal do OSIQ, segundo sexo e fase inicial e intermediária da adolescência. São Bernardo do Campo (SP), 2006

Table 5. Summary of findings of validity and reliability of body image subscale of OSIQ, by sex and phase (early or late) of adolescence. São Bernardo do Campo (SP), 2006

\begin{tabular}{|c|c|c|c|c|c|c|c|c|c|}
\hline \multirow[b]{2}{*}{ Análises } & \multirow[b]{2}{*}{ Parâmetro } & \multicolumn{4}{|c|}{ Masculino } & \multicolumn{4}{|c|}{ Feminino } \\
\hline & & $\begin{array}{l}\text { Adolescência } \\
\text { inicial } \\
\text { (10-14 anos) }\end{array}$ & $\mathrm{p}$ & $\begin{array}{c}\text { Adolescência } \\
\text { intermediária } \\
(15-18 \text { anos) }\end{array}$ & $\mathrm{p}$ & $\begin{array}{l}\text { Adolescência } \\
\text { inicial } \\
\text { (10-14 anos) }\end{array}$ & $\mathrm{p}$ & $\begin{array}{c}\text { Adolecência } \\
\text { intermediária } \\
\text { (15-18 anos) }\end{array}$ & $\mathrm{p}$ \\
\hline \multicolumn{10}{|c|}{ Validade discriminante média $(\mathrm{dp})$} \\
\hline magreza & & $35,6(5,6)$ & 0,043 & $31,5(4,3)$ & 0,100 & $31,7(7,5)$ & 0,133 & - & 0,23 \\
\hline peso normal & & $32,5(5,3)$ & & $34,1 \quad(3,9)$ & & $30,3(4,6)$ & & $30,56(5,0)$ & \\
\hline sobrepeso & & $29,4(6,7)$ & & $30,3(5,5)$ & & $27,5(5,7)$ & & $28,56(4,5)$ & \\
\hline obeso & & $28,2(5,7)$ & & $30,0(6,2)$ & & $25,2(6,9)$ & & $27,60(3,9)$ & \\
\hline Validade Concorrente & $r(p)$ & & & & & & & & \\
\hline IMC & & $-0,12$ & 0,202 & $-0,06$ & 0,657 & $-0,23$ & 0,008 & $-0,02$ & 0,874 \\
\hline RCQ & & $-0,16$ & 0,093 & $-0,19$ & 0,130 & $-0,07$ & 0,436 & $-0,29$ & 0,010 \\
\hline CC & & $-0,13$ & 0,177 & $-0,10$ & 0,424 & $-0,26$ & 0,003 & $-0,04$ & 0,717 \\
\hline Consistência Interna & $\alpha$ Cronbach & 0,52 & & 0,54 & & 0,43 & & 0,49 & \\
\hline \multirow[t]{3}{*}{ Confiabilidade } & média (dp) & $32,2(5,5)^{\mathrm{M} 1}$ & 0,090 & $33,2(4,6)^{\mathrm{M} 1}$ & 0,198 & $30,1(5,1)^{\mathrm{M} 1}$ & 0,829 & $30,1(4,9)^{\mathrm{M} 1}$ & 0,044 \\
\hline & & $33,0(6,0)^{\mathrm{M} 2}$ & & $33,9(5,1)^{\mathrm{M} 2}$ & & $30,1(6,1)^{\mathrm{M} 2}$ & & $30,9(5,2)^{\mathrm{M} 2}$ & \\
\hline & $r_{\text {jsc }}(p)$ & 0,65 & $<0,001$ & 0,45 & $<0,001$ & 0,72 & $<0,001$ & 0,46 & $<0,001$ \\
\hline Tempo (segundos) & média (dp) & $0,91(0,65)$ & & $0,76(0,42)$ & & $1,01(0,46)$ & & $0,86(0,48)$ & \\
\hline Compreensão verbal & média $(\mathrm{dp})$ & $3,3(1,1)$ & & $3,2(1,5)$ & & $3,4(0,9)$ & & $3,3(1,4)$ & \\
\hline
\end{tabular}

IMC: índice de massa corporal; RCQ: razão circunferência cintura/quadril; CC: circunferência da cintura, (dp): desvio-padrão; $r$ (p): correlação; $r_{\text {icc }}(p)$ : correlação intraclasse; ${ }^{\text {M1 Momento } 1 ;{ }^{\text {M2}} \text { Momento } 2}$

IMC: body mass index; RCQ: waist/hip circumference ratio; CC: waist circumference; (dp): standard deviation: $r(p)$ : correlation; $r_{\text {icc }}(p)$ : interclass correlation coefficient; ${ }^{T 1}$ Time 1, ${ }^{\text {T2Time } 2}$ 


\section{Discussão}

O OSIQ, desde sua primeira versão, em 1961, caracterizou-se por pesquisar as diferenças entre os adolescentes com desenvolvimento normal e aqueles com desenvolvimento comprometido. As novas versões passaram por ajustes, com inclusão e retirada de itens. $\mathrm{O}$ instrumento aplicado nesta pesquisa refere-se à última versão do OSIQ ${ }^{5}$, publicada no ano de 1992, contendo, ao todo, sete itens para a avaliação da subescala de imagem corporal.

No presente estudo, apreciou-se apenas a equivalência semântica e de mensuração da subescala de imagem corporal do OSIQ, embora um processo de adaptação transcultural formal deva considerar as seguintes etapas: equivalências conceitual, de item, semântica, operacional, mensuração e funcional ${ }^{10,11}$. Sendo assim, os dados aqui apresentados são ainda insuficientes para que se possa ter uma avaliação global acerca da equivalência dessa subescala para uso em nosso meio.

Observou-se na primeira etapa, que somente a expressão fairly well mereceu maior atenção, exigindo sua tradução para "mais ou menos", de forma a preservar o seu significado referencial ${ }^{11}$. Ainda que a versão inicial tenha sido pré-testada antes do trabalho de campo, obtendo-se
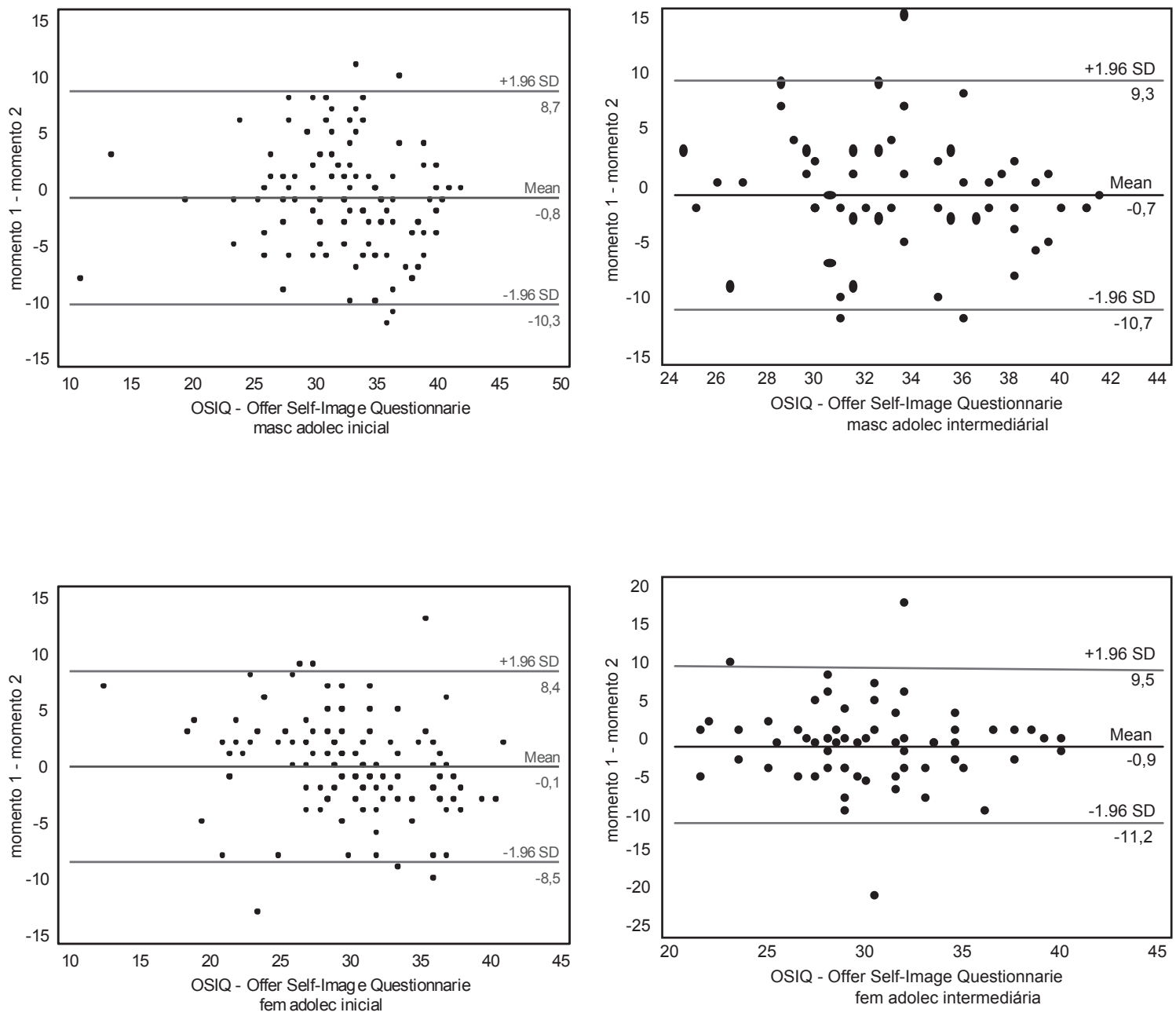

Figura 1. Gráficos de Bland e Altman da subescala de imagem corporal do OSIQ para as populações masculina e feminina, nas fases inicial e intermediária de adolescência. São Bernardo do Campo (SP), 2006

Figure 1. Bland and Altman plots of body image subscale of OSIQ for male and female subjects in early and middle phases of adolescence. São Bernardo do Campo (SP), 2006 
resultados satisfatórios, essa etapa foi realizada com um número reduzido de jovens. Já na aplicação do instrumento, observou-se que a escala gerou dúvidas, pois os adolescentes expressaram dificuldade em estabelecer a relação entre uma das seis opções de respostas em cada item. Outro aspecto que não foi bem compreendido, observado durante a aplicação, foi em relação à questão 7 , com o termo "sou pouco desenvolvido". Os jovens revelaram dificuldade em compreender o significado dessa expressão, que se trata, de acordo com a versão original ${ }^{5}$, do tema "apresentação corporal", ou seja, do tamanho corporal. Esse aspecto, provavelmente, deve-se ao fato de o instrumento não ter sido avaliado por especialistas da área de desenvolvimento humano conforme sugerido por Herdman e Fox-Rushby e Badia ${ }^{10}$ e Hasselmann e Reichenheim ${ }^{11}$, recebendo atenção somente de três profissionais de área de conhecimento distinta (Estatística, Saúde da Família e Psicologia). Todos esses fatos podem ter influenciado na qualidade da versão final do instrumento.

Cabe destacar a escolha feita na metodologia do critério aplicado para a apreciação da equivalência de mensuração. Nos estudos envolvendo esse tema, é usual a aplicação de medidas de peso e $\mathrm{IMC}^{3,4,18}$ como medidas de comparação. Sendo assim, por não haver um padrão ouro para avaliar a imagem corporal, considerou-se que adolescentes com sobrepeso e obesidade inferem um maior grau de insatisfação, tendo sido este o critério utilizado para avaliar tanto a validade discriminante quanto a concorrente.

Smolak ${ }^{19}$ afirmou que grande parte dos estudos e da produção científica, com enfoque no desenvolvimento infantojuvenil, deu prioridade ao aspecto "satisfação corporal", associando-o a questões como idade, sexo, estado nutricional, qualidade da relação familiar, pressão social, entre outros. Entre os pesquisadores da área, há o seguinte consenso: os adolescentes com sobrepeso e obesidade, de ambos os sexos, apresentam maior insatisfação quando comparados a seus pares ${ }^{20,21}$.
Outro ponto importante a ser mencionado refere-se às características da população estudada. A amplitude da faixa etária, ou seja, dos 10 aos 18 anos, engloba diferentes estágios de cognição e maturação sexual. Embora o instrumento tenha sido elaborado para adolescentes, e no estudo original esse aspecto não tenha sido destacado, observou-se, na população mais jovem, certa dificuldade para a compreensão da escala. Sendo assim, para aprofundar esse ponto, optou-se também pela realização da análise estatística estratificada, segundo as seguintes fases da adolescência: inicial - 10 a 14 anos - e intermediária - 15 a 18 anos.

$\mathrm{Na}$ análise da consistência interna para os sexos masculino e feminino, englobando as duas fases da adolescência, ou seja, inicial e intermediária, os valores variaram de 0,43 a 0,54 . Laukkanes et al. ${ }^{22}$, ao aplicarem o OSIQ ${ }^{5}$ completo, obtiveram um coeficiente $\alpha$ de Cronbach de 0,80. Os baixos valores relacionam-se, provavelmente, à presença de dúvidas no preenchimento do questionário e também ao fato de a escala ter sido aplicada isoladamente em relação às demais escalas que compõem o OSIQ. A comparação dos resultados com os estudos internacionais deve ser realizada com certa ponderação, pois não há a mesma padronização na aplicação e análise. Os dados disponíveis referem-se à aplicação do OSIQ na íntegra, o que não ocorreu no presente estudo. Mesmo assim, observa-se que a consistência interna do instrumento não respondeu preservando a coerência e integração dos itens que compõem o construto imagem corporal. Dessa forma, percebeu-se que não houve diferença nos resultados obtidos entre os jovens em relação à idade.

$\mathrm{Na}$ apreciação da validade discriminante, a escala foi capaz de discriminar entre os quatro grupos estudados (magro, peso normal, sobrepeso e obesidade) para a população total, meninos e meninas. Interessante observar que pela análise, segundo fases da adolescência, somente para meninos em fase inicial, a escala apresentou resultado satisfatório, ou seja, foi 
capaz de discriminar entre os grupos estudados. O mesmo resultado não foi observado para os meninos na fase intermediária e meninas nas fases inicial e intermediária. Sendo assim, para esses jovens, não há diferença entre o grau da satisfação corporal e sua avaliação da imagem corporal. Para as meninas, essa característica é bem conhecida, ou seja, meninas são mais exigentes e insatisfeitas em relação à sua corporeidade. Esse fato pode ser evidenciado pela análise estratificada. Já para os meninos em fase intermediária, este resultado, provavelmente, relaciona-se, de modo igual ao aumento de exigência na apresentação corporal. Mesmo assim, os resultados das análises, de acordo com o sexo e a população total devem ser valorizados, pois comprovaram que a escala foi capaz de discriminar os grupos pesquisados, de acordo com estas condições.

Laukkanes et al. ${ }^{22}$, mesmo com a aplicação de outros instrumentos de comparação, desenvolveram estudo de validade discriminante das 12 subescalas do OSIQ com adolescentes finlandeses. Os autores concluíram que o OSIQ ${ }^{5}$ pode ser aplicado como um método de triagem para adolescentes com desenvolvimento normal. Já Petersen et al. ${ }^{7}$ estudaram uma versão abreviada do OSIQ: o Self-Image Questionnaire for Young Adolescent (SIQYA) e desenvolveram análise discriminante com adolescentes. Constataram, para a escala de imagem corporal, associação estatisticamente significativa para os adolescentes que relataram comprometimento para depressão e problemas escolares, inferindo imagem corporal negativa.

Observou-se, como esperado no presente estudo, na apreciação da validade discriminante, que adolescentes com sobrepeso e obesidade expressam maior insatisfação em relação à imagem corporal. Esta característica foi confirmada para análise segundo sexo.

Já na apreciação da validade concorrente, houve correlação significativa entre as três medidas (IMC, RCQ e CC) somente para o sexo feminino. Para os meninos, foi possível a confirmação da correlação para a RCQ e, para a população total, as medidas de IMC e CC. Para esta análise, observou-se que as meninas com sobrepeso e obesidade expressaram maior insatisfação em relação a seus pares, contudo o mesmo não foi observado para os meninos. Mas, observando-se a análise segundo a fase da adolescência somente para as adolescentes - fase inicial (IMC e CC) e intermediária (RCQ) foi possível confirmar a correlação para as medidas antropométricas. Uma possível explicação para esta diferença refere-se aos fatores relacionados à insatisfação corporal dos adolescentes. É sabido que meninas são mais insatisfeitas e preocupadas com peso ${ }^{23}$ e meninos preocupam-se em aumentar o tamanho e músculos ${ }^{24}$. Sendo assim, levando-se em consideração o sexo masculino, a avaliação da imagem corporal dos meninos não se diferenciou em relação ao IMC e à CC. Já considerando-se a fase da adolescência, essa característica não fica evidenciada; ou seja, meninos em fase inicial e intermediária apresentam valores semelhantes de insatisfação.

Confirma-se, assim, a hipótese para o OSIQ, na apreciação da validade discriminante para os sexos masculino, feminino e para meninos em fase inicial de adolescência e na apreciação da validade concorrente, com as três medidas, somente para o grupo de meninas.

Vale ressaltar os resultados satisfatórios da subescala de imagem corporal do OSIQ em relação à validade discriminante e concorrente. $\mathrm{O}$ instrumento foi capaz de discriminar os subgrupos estudados, de acordo com o estado nutricional e pode correlacionar-se com as medidas antropométricas.

Já em relação à confiabilidade, entre os momentos 1 e 2, a subescala de imagem corporal do OSIQ apresentou igualdade na comparação das médias somente para o grupo das meninas e dos meninos em fases incial e intermediária da adolescência, embora as estimativas pontuais das médias para os demais grupos sejam muito próximas. Já na correlação intraclasse, os 
valores foram inferiores a 0,65 para os grupos estudados.

Nos estudos internacionais, dados de confiabilidade não são descritos, o que inviabiliza qualquer comparação entre os achados da presente pesquisa com os mesmos.

O tempo médio de aplicação da escala do OSIQ foi bom, inferior a dois minutos. Já para a compreensão verbal, os valores médios foram de 3,3 minutos (máximo=4,0), demonstrando ser de rápida aplicação e razoável compreensão.

Sendo assim, observa-se que as características psicométricas da subescala de imagem corporal do OSIQ, apesar de confirmar a hipótese na apreciação da validade discriminante para meninos e meninas, na apreciação da validade concorrente para meninas, e bons valores na compreensão verbal e tempo de aplicação, não apresentou resultados satisfatórios para consistência interna e confiabilidade, não preservando a coerência e integração dos itens que compõem o construto imagem corporal.

Novos estudos sobre o tema são necessários, levando-se em consideração os baixos valores da consistência interna. Sugere-se novo procedimento de apreciação de equivalência semântica, dando especial atenção às dificuldades observadas na presente pesquisa. Dessa forma, o instrumento apresenta-se traduzido, mas carece de futuros estudos com enfoque na adaptação transcultural e equivalência de mensuração, a fim de que haja melhor adequação à realidade brasileira.

\section{Referências}

1. Slade PD. What is body image? Behav Res Ther. 1994;32(5):497-502.

2. Cash TF, Pruzinsky T. Body image: a handbook of theory, research, and clinical practice. New York: The Guilford Press; 2002.

3. Thompson JK. The (mis)measurement of body image: ten strategies to improve assessment for applied and research purposes. Body Image. 2004; 1(1):7-14.

4. Mendelson BK, Mendelson MJ, White DR. Body-steam scale for adolescents and adults. J Pers Assess. 2001;76(1):90-106.

5. Offer D, Ostrov E, Howard KI, Dolan S. A manual for the Offer Self-Image Questionnaire for adolescents (OSIQ). Chicago: Michael Reese Hospital, 1982.

6. Lindfors K, Elovainio M, Sinkkonen J, Aalberg V, Vuorinen R. Construct validity of the Offer Self-Image Questionnaire and its relationship with self-esteem, depression, and ego development. J Youth Adolesc. 2005;34(4):389-400.

7. Petersen AC, Schulenberg JE, Abramowitz RH, Offer D, Jarcho HD. A Self- Image Questionnaire for Young Adolescents (SIQYA): Reliability and Validity Studies. J Youth Adolesc. 1984;13(2):93-111.

8. Erkolahti R, Jansson J, Offer D, Steinhausen HC. Comparison of the self-image of teenagers in Finland, the United States and Germany. J Adolesc Health. 1992;13(5):392-395.
9. Laukkanen ER, Shemeikka SL, Viinamäki, Pölkki PL, Lehtonen JO. Heavy drinking is associated with more severe psychosocial dysfunction among girls than boys in Finland. J Adolesc Health. 2001;28(4):270-7.

10. Herdman M, Fox-Rushby J, Badia X. A model of equivalence in the cultural adaptation of HEQoL instruments: the universalist approach. Qual Life Res. 1998;7(4):323-35.

11. Hasselmann MH, Reichenheim ME. Adaptação transcultural da versão em português das "Conflict Tactics Scales Form R" (CTS1) usada para aferir violência no casal: equivalência semântica e de mensuração. Cad. Saúde Pública. 2003;19(4):1083-93.

12. Grassi-Oliveira R, Stein LM, Pezzi JC. Tradução e validação de conteúdo de versão em Português de Childhood trauma questionnaire. Rev Saúde Pública. 2006;40(2):249-55.

13. Polce-Lynch M, Myers BJ, Kliewer W, Kilmartin C. Adolescent self-esteem and gender: exploring relation to sexual harassment body image, media influence, and emotion expression. J Youth Adolesc. 2001;30(2):225-244.

14. Gordon CC, Chumlea WC, Roche AF. Stature, recumbent length, and weight. In: Lohman TG, Roche AF, Martorell R. Anthropometric standardization reference manual. Champaing: Human Kinetics Books; 1988.

15. Heyward VH, Stolarczzyk LM. Avaliação da composição corporal aplicada. São Paulo: Manole; 2000. 
16. [WHO] World Health Organization. Physical status: the use and interpretation of anthropometry. WHO Techinical Reports Series, 854. Genova: WHO; 1995.

17. Bland JM, Altman DG. Statistical methods for assessing agreement between two methods of clinical measurement. Lancet. 1986;1(8476):307-310.

18. Wertheim EH, Paxton SJ, Tilgner L. Test-retest reliability and construct validity of contour drawing rating scale scores in a sample of early adolescent girls. Body Image. 2004;1(2):199-205.

19. Smolak L. Body image in children and adolescent: where do we go from here? Body Image. 2004;1(1):15-28.

20. Gardner RM. Body image assessment of children. In: Cash TF, Pruzinsky T. Body image: a handbook of theory, research, and clinical practice. The Guilford Press: New York; 2002.

21. Thompson JK, Gardner RM. Measuring perceptual body image among adolescents and adults. In: Cash TF. Pruzinsky
T. Body image: a handbook of theory, research, and clinical practice. New York: The Guilford Press; 2002.

22. Laukkanen ER, Peiponen S. Halone P, Aivio A, Viinamäki H. Discriminant validity of the Offer Self-Image Questionnaire in finís 13-year-old adolescents. Nord J Psychiatry. 1999;53:197-201.

23. Smolak L, Levine MP, Thompson JK. The use of the Sociocultural Attitudes Towards Appearance Questionnaire with middle school boys and girls. Int J Eat Disord. 2001;29(2):216-23.

24. McCabe MP, Ricciardelli LA. A longitudinal study of pubertal timing and extreme body change behaviors among adolescent boys and girls. Adolescence. 2004;39(153):145-66.

Recebido em: 08/08/2008 Versão final apresentada em: 27/01/2011

Aprovado em: 19/04/2011 